\title{
Tracheobronchial tear in chest trauma-a diagnostic perplexity
}

\author{
Guruprasad Rai ${ }^{1}$ (D) Ganesh Sevagur Kamath ${ }^{1} \cdot$ Rajkamal Vishnu $^{1}$. \\ Shaheem Shanavas ${ }^{1}$
}

Received: 29 August 2017 / Revised: 17 October 2017 / Accepted: 19 October 2017 / Published online: 13 November 2017

(C) Indian Association of Cardiovascular-Thoracic Surgeons 2017

\begin{abstract}
Tracheobronchial injuries following blunt chest trauma are uncommon and diagnosed late. Tracheal rupture due to a fall from a height is rare. Early diagnosis and prompt treatment give gratifying results. We present a case of a $42-$ year-old man who was referred at our hospital with alleged history of fall from a height of about $12 \mathrm{ft}$ from a tree. On presentation, the patient was haemodynamically stable and right-sided ICT was already inserted. There was a minimal surgical emphysema in the neck, a continuous air leak from the chest tube and a partially collapsed right lung on chest Xray. After a few hours, he desaturated and was intubated. The right lung did not expand in spite of the second apical chest tube, and on ventilation, air leak increased. Tracheabronchial tear was suspected and computerised tomography scan revealed the site of damage and fibreoptic bronchoscopy confirmed the diagnosis. Emergency thoracotomy with primary repair of the right tracheabronchial tear was done. The patient recovered and post-operative course was uneventful.
\end{abstract}

Keywords Intercostal tube (ICT) · Tracheobronchial tear · Early repair

\section{Introduction}

The first reported case of traumatic ruptured bronchus is attributed to Webb in 1848 following a post-mortem on a man run over by a cart [1]. Primary surgical repair was first

Guruprasad Rai

drgururai13@gmail.com

1 Department of CardioThoracic Surgery, Kasturba Medical College, Manipal University, Manipal, Udupi, Karnataka, India successfully performed by Scannell in 1951 [2]. Over $80 \%$ of injuries occur within $2 \mathrm{~cm}$ of the carina and most are in young patients with relatively elastic chest walls. Presenting symptoms vary depending on the severity and mechanism of injury. In severe cases, it is almost always fatal; however, in milder cases, the diagnosis can often be missed owing to absence of classical features. The outcome is mainly dependent on the timing of diagnosis and associated injuries. A delayed diagnosis often results in long-term complications which eventually require resection of the lung, but there are reports where delayed surgery also gave good results [3]. Two clinical presentations have been documented, depending on whether the peribronchial tissues remain intact. Complete tear results in pneumothorax, surgical emphysema, non-expansile lung and air leak worsening with placement of an ICT. The diagnosis in this case of complete rupture is usually obvious and patient requires urgent intervention. When the peribronchial tissues are intact, no air leak occurs, the lung does not collapse and diagnosis is missed. The patient recovers on conservative management.

\section{Case report}

A 42 year-old man with no pre-morbidities but under the influence of alcohol suffered blunt injury to his chest as he fell down from a tree of around $12 \mathrm{ft}$ height at 6 p.m. in his village. There was no history of loss of consciousness, seizures or any episodes of vomiting. He was immediately taken to a local hospital as he complained of severe right-sided chest pain and breathing difficulty. ICT was inserted for pneumothorax on the right side and an air leak was noticed. He was transferred to our institution with a chest tube in situ for further management. On arrival in the Trauma Triage at 8.30 p.m., the patient was conscious and oriented with stable vitals, 
maintaining oxygen saturation at room air; subcutaneous emphysema was noted over the chest wall and supraclavicular region on the right side. Chest radiograph showed a collapsed right lung with the chest tube, with no rib fracture; continuous air bubbling was noted in the pleural drainage. An urgent ultrasound in the abdomen ruled out any intra-abdominal injuries. The patient was admitted under the Department of General Surgery and was kept under watchful observation in surgical intensive care. A few hours later at around $5 \mathrm{am}$, the patient complained of severe dyspnoea and desaturated; he was intubated and mechanically ventilated. He remained hypoxic despite intubation and the air leak further increased; hence, cardiothoracic surgeon opinion was taken. The resident inserted one more apical ICT to expand the lung but of no avail (Fig. 1). CT scan thorax was done immediately and showed a doubtful flap in the lower trachea before bifurcation on the right side and near total collapse of the distal right main bronchus with air lucencies around the bronchus (Fig. 2). A presumptive diagnosis of injury to the right tracheobronchial tract was made. The patient was immediately taken up for a surgery at 8 a.m. (14 h after the fall) and was stabilised with left endobronchial tube intubation. Next, a paediatric fibreoptic bronchoscope was passed through the tracheal opening of the endobronchial tube which revealed severe inflammation at the right main bronchus origin with mucoid material; the lateral part of the tracheobronchial junction was open and the pleural cavity and the collapsed lung could be seen. The tear was of approximately $4 \mathrm{~cm}$ vertically; on further probing, the scope entered into the intermediate bronchus which was normal, and laterally, the collapsed origin of the upper lobe was seen and the middle lobe and lower lobe origins were normal with mucoid material. The patient was positioned left full lateral and with right posterolateral thoracotomy, the chest entered through the fourth intercostal space. There were purulent flakes all over; the lung was collapsed and was not ventilating; the tear identified at the right tracheabronchial junction and surrounding tissues was dissected to expose the opening, after ligation of the azygous vein.
The zigzag-shaped tear commenced about $4 \mathrm{~cm}$ above the origin of the right main bronchus; it made a V-shaped flap of the tracheal cartilage and the right main bronchus was partially transected (Fig. 3). The left endobronchial tube was seen through the opening. The tracheobronchial tear was repaired with 2-0 Ethibond ${ }^{\circledR}$ interrupted sutures. All interrupted sutures were taken initially and tied at the end. First, a horizontal mattress suture was taken at the tip of the V-shaped flap and was pulled so that the rest of sutures could be taken easily. The right tracheobronchial angle, carina and intermediate bronchus area were already dissected to obtain more space and mobility. The margins approximated easily when the sutures were pulled and tied sequentially. The fifth intercostal muscle was mobilised as a pedicle from the line of anterior axillary line and dissected till the para vertebral area and the intercostal artery were clipped at the tip. This pedicle was used to cover the suture line over the tracheobronchial angle with $5-0$ Prolene ${ }^{\circledR}$ sutures all around the sutured part of the trachea. The presence of purulent flakes in the pleural cavity prompted us to use this measure as a prophylaxis against infection. On carefully re-expanding the right lung, no air leak was noted. Immediate post-operative chest radiograph showed the fully expanded right lung. After being mechanically ventilated for 2 days, he was weaned off the ventilator. There was no air leak noted in chest drains; the patient recovered well; however, on post-operative day 12 , he was found to have surgical site infection which required secondary suturing. He was discharged from the hospital 20 days after the trauma.

\section{Discussion}

Tracheobronchial rupture is defined as any injury to the trachea or bronchi which is localised between the cricoid cartilage and the division of lobar bronchi into their segmental bronchi. It can result due to severe blunt trauma, penetrating or gun-shot injuries and iatrogenic. Tracheobronchial injuries following blunt trauma are exceedingly rare but can be life-
Fig. 1 Chest X-ray. a Lateral ICT inserted in previous hospital. b After insertion of apical anterior ICT
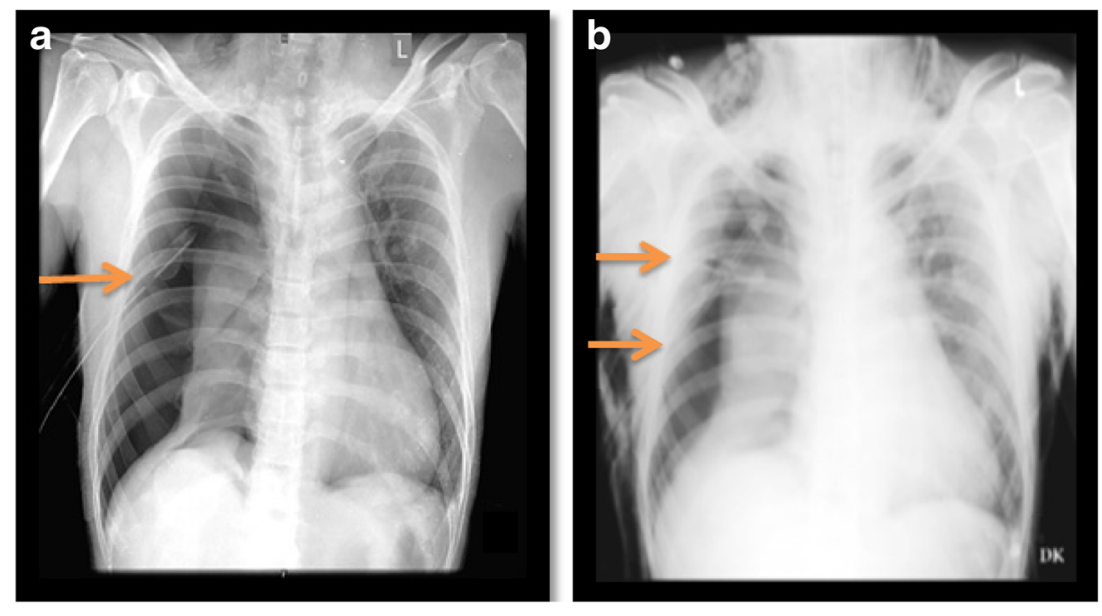
Fig. 2 CT thorax - the collapsed right lung with collapse of the distal main bronchus

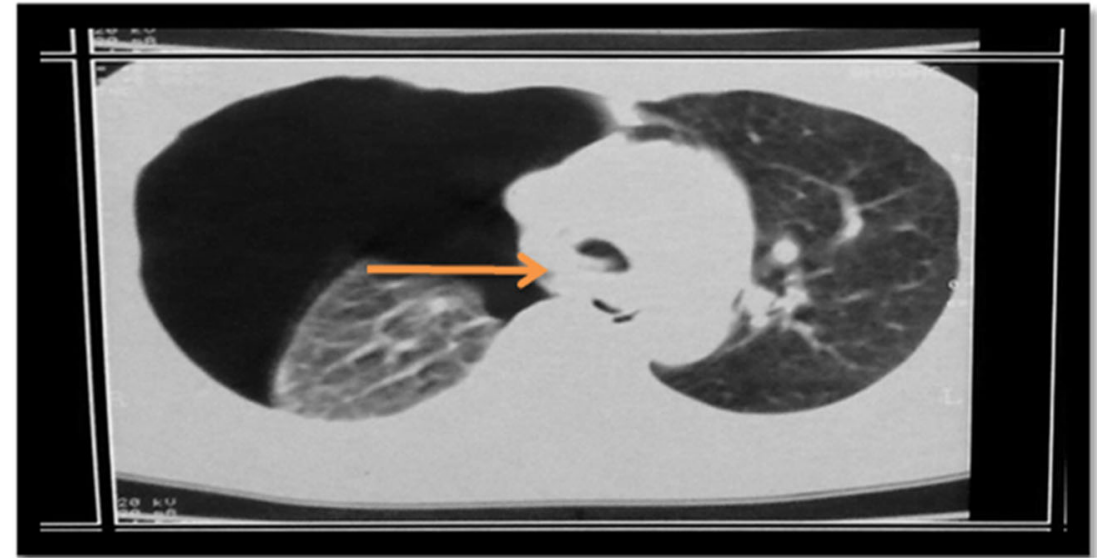

threatening [4]. It warrants accurate pre-operative diagnosis and earliest possible restoration of continuity of air passages. The pathophysiologic basis of a bronchial rupture includes three mechanisms: a decrease in the anteroposterior diameter of the thorax, sudden increase in intrabronchial pressure with a closed glottis and rapid deceleration or compression between the sternum and the vertebral column or torsion of the tracheobronchial tree [5]. Injuries are not diagnosed immediately in 25 to $68 \%$ of patients [6]. Two distinct clinical presentations may occur, first with free communication between the bronchus and the pleura and second with little or no communication. The first instance, there is a complete tear, and in the second presentation, the peribronchial tissues are intact. In the presence of complete tear, air will leak into the pleural space and mediastinum resulting in pneumothorax and surgical emphysema. The lung will remain collapsed. When the peribronchial tissues are intact then patient will only have haemoptysis and pain. There is possibility that the peribronchial tissue may give away after $8-10$ days and then the patient will develop pneumothorax and surgical emphysema as a late onset phenomenon. In this late presentation, the morbidity is more as the tissues are very friable and may require lung resection. Bronchoscopy is the gold standard for the confirmation of diagnosis and should be carried out even in suspicious cases [7]. Tracheobronchial injury following blunt chest trauma was first described in 1912 by Schonberg. The first successful delayed repair of a tracheobronchial injury was performed by Krinitzki in 1949 [1]. He successfully anastomosed a ruptured bronchus 2 months after the injury. Scannell in 1951 was the first surgeon to perform an immediate primary repair of tracheobronchial injury following closed chest injury [2]. Presenting symptoms vary depending on the severity and mechanism of injury. In severe cases, it is almost always fatal; however, in milder cases, the diagnosis can often be missed owing to absence of classical features. The outcome is mainly dependent on the timing of diagnosis and associated injuries. A delayed diagnosis often results in complications and resection of the lung and mortality $[8,9]$. Glazer and colleagues in their review of tracheobronchial injuries have shown excellent results even with delayed presentation [3].

In our case, the surgical repair was the option made on an emergency basis, taking into account the clinical picture, roentgenogram, CT scan and deteriorating oxygen saturations of the patient and then fibreoptic bronchoscope confirmation. The bronchial rupture was within $2 \mathrm{~cm}$ of origin of the right main bronchus, a classic site described in literature. The
Fig. 3 Pictorial representation. a Illustrative diagram of the tear. $\mathbf{b}$ Visual representation of the zigzag-shaped tear above the origin of the right main bronchus

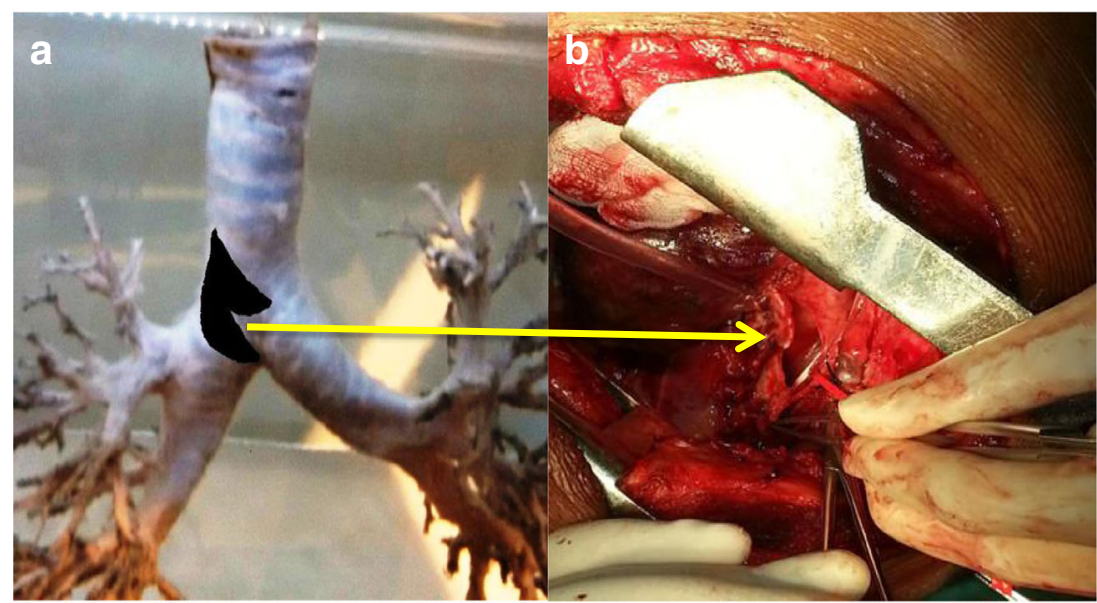


precaution a surgeon should take is to prevent any tension at the suture line and the distance between sutures should be closed without any gaps. The whole area should be properly mobilised from the surrounding tissues, and if required, sometimes, the inferior pulmonary ligament should be incised to lift up the lung. Total devascularisation should not be done as blood supply will be compromised.

\section{Conclusion}

Tracheobronchial injuries are rare due to blunt trauma caused by a fall from a short height. Diagnosis can be easily missed and treatment can be delayed. A strong clinical suspicion aided with proper interpretation of the clinical findings, investigations and bronchoscopy confirms the diagnosis. Bronchoscopy is the gold standard and one should have a low threshold to perform it in case of doubt.

Learning points from this case are, in any case of chest trauma with pneumothorax and surgical emphysema, if the lung does not expand after the chest tube and the chest drainage has continuous bubbling, the patient has to be investigated immediately for tracheabronchial injuries and not wait till the patient becomes unstable and hypoxic. Computerised tomography scan and fibreoptic bronchoscopy are mandatory at this point. With improvement in anaesthetic and surgical techniques, the prognosis of tracheobronchial rupture is excellent and patient can lead a normal life after surgery.

\section{Compliance with ethical standards}

Informed consent Informed consent was taken.
Conflicts of interest The authors declare that they have no conflict of interest.

Statement of human and animal rights The procedures followed were in accordance with the ethical standards of the responsible committee on human experimentation (institutional and national) and with the Helsinki Declaration of 1975, as revised in 2000 and 2008.

\section{References}

1. Roxburg JC. Rupture of the tracheobronchial tree. Thorax. $1987 ; 42$ : $681-8$.

2. Scannell JG. Rupture of the bronchus following closed injury to the chest. Ann Surg. 1951;133:127-30.

3. Glazer ES, Meyerson SL. Delayed presentation and treatment of tracheobronchial injuries due to blunt trauma. J Surg Educ. 2008;65:302-8.

4. Jones WS, Mavroudis C, Richardson DJ, Gray LA Jr, Howe WR. Management of tracheobronchial disruption resulting from blunt trauma. Surgery. 1984;95:319-23.

5. Kirsh MM, Orringer MB, Behrendt DM, Sloan H. Management of tracheobronchial disruption secondary to non-penetrating trauma. Ann Thorac Surg. 1976;22:93-101.

6. Symbas PN, Justicz AG, Ricketts RR. Rupture of the airways from blunt trauma: treatment of complex injuries. Ann Thorac Surg. 1992;54:177-83.

7. Becmeur F, Donato L, Horta-Gerand P, et al. Rupture of the airways after blunt chest trauma in two children. Eur J Pediatr Surg. 2000;10: $133-5$.

8. Deslauriers J, Beaulieu M, Archambault G, LaForge J, Bernier R. Diagnosis and long term follow up of major bronchial disruption due to non-penetrating trauma. Ann Thorac Surg. 1982;33:32-9.

9. Kiser AC, O'Brien SM, Detterbeck FC. Blunt tracheobronchial injuries: treatment and outcomes. Ann Thorac Surg. 2001;71:2059-65. 